\title{
REVIEW \\ Can combined aerobic and muscle strength training improve aerobic fitness, muscle strength, function and quality of life in people with spinal cord injury? A systematic review
}

\author{
V Bochkezanian ${ }^{1}$, J Raymond ${ }^{2}$, CQ de Oliveira $^{2}$ and GM Davis ${ }^{2}$
}

Study design: A systematic review.

Objectives: The aim of this systematic review was to establish whether combined aerobic training and muscle strength training is effective in improving aerobic fitness, muscle strength, function and/or quality of life (QoL) in people with spinal cord injury (SCl).

Settings: Faculty of Health Sciences. University of Sydney, NSW, Australia.

Methods: A search was conducted for randomized controlled trials (RCTs), controlled trials, uncontrolled clinical trials, case series and cross-over studies involving exercise interventions that included a combination of aerobic and strength components, either in circuitmode or in sequence for people with SCI. Methodological quality was independently rated using the PEDro scale and key findings were extracted from trials by two reviewers.

Results: The search identified 7981 abstracts, from which nine trials met the inclusion criteria. From the nine selected trials, seven reported aerobic outcomes, two of which showed a statistically significant within-group difference in aerobic fitness. Five studies reported muscle strength outcomes, four of them showed a statistically significant within-group mean difference on at least one outcome measure. Two studies looked at QoL, one of them found a statistically significant between-group difference on one outcome measure.

Conclusion: Our systematic review showed that literature on $\mathrm{SCI}$ population is scarce, of low quality and findings of existing studies are inconsistent. Thus, further RCTs with larger number of participants are needed to make a definite conclusion about the influence of combined aerobic and muscle strength training on aerobic fitness, muscle strength and QoL in people with SCl.

Spinal Cord (2015) 53, 418-431; doi:10.1038/sc.2015.48; published online 31 March 2015

\section{INTRODUCTION}

\section{Rationale}

A spinal cord injury (SCI) leads to dramatic changes in a person's lifestyle. As a result of a spinal cord lesion, physical impairments arise that have a negative impact on the ability of the individual to perform activities within the range considered 'normal' for a human being, consequently diminishing physical functioning (that is, the execution of a task, such as transfer from the wheelchair to the bed) and quality of life (QoL) $\cdot{ }^{1-3}$ Exercise interventions are widely available that deal with two important physical impairments: low aerobic fitness and reduced muscle strength and endurance.

Aerobic fitness is reduced after SCI due to impaired redistribution of arterial blood, venous blood pooling below the level of the spinal cord lesion (that is, deep venous thrombosis), ${ }_{4}^{4}$ altered cardiovascular autonomic control ${ }^{4}$ and a more sedentary lifestyle ${ }^{5}$ in comparison to able-bodied people. ${ }^{6}$ Muscle strength is decreased because neurological input to the affected muscles that retain partial innervation is deficient, leading to the loss of normal activation timing and force-generating capacity. ${ }^{7}$ Secondary to the loss of neural inputs are negative musculoskeletal adaptations such as transformation to faster muscle fibers (IIa, IIb) ${ }^{7}$ increased contractile speed and increased fatigue in partially paralyzed and also innervated muscles in people with SCI. ${ }^{7}$ Moreover, lack of training of innervated muscles may compromise physical function resulting in physical and QoL impairments. ${ }^{89}$ By improving these physical impairments (that is, low aerobic fitness and reduced muscle strength to partial innervated and innervated regions), exercise interventions aim to impact positively on the functional outcomes and QoL, becoming the ultimate goal of post-traumatic rehabilitation. ${ }^{10-12}$ However, finding an intervention that is effective in targeting both muscle strength and aerobic fitness, and one that can be performed in a short period of time is challenging.

Combined aerobic and strength conditioning is potentially useful because it can improve both impairments, as well as improve functional outcomes in a relatively short period of time. Such combined-mode training may also be cost effective, as it can be performed in any community gym, that is adapted to wheelchair use. Previous studies that have investigated this type of intervention demonstrated improvements in aerobic fitness and/or muscle strength. ${ }^{13-18}$ In addition, some previous reports have found that combined-mode interventions may also be effective for improving functional abilities and QoL. ${ }^{11,14-16,18,19}$ Nevertheless, the findings of these previous investigations have not been subject to the rigors of

${ }^{1}$ Faculty of Health, Engineering and Science, Edith Cowan University, Joondalup, WA, Australia and ${ }^{2}$ Faculty of Health Sciences, The University of Sydney, Sydney, NSW, Australia Correspondence: V Bochkezanian, Faculty of Health, Engineering and Science, School of Exercise and Health, Edith Cowan University, Building 21, Office 501, Joondalup Campus, 270 Joondalup Drive, Joondalup, WA 6027, Australia.

E-mail: v.bochkezanian@ecu.edu.au

Received 21 July 2014; revised 22 January 2015; accepted 23 February 2015; published online 31 March 2015 
systematic review or meta-analysis techniques to arrive at a definite conclusion as to their physiological benefits or clinical efficacy. Although many systematic reviews have analyzed physical fitness in people with SCI, these have encompassed any general physical exercise interventions, including either aerobic fitness or muscle strength training in isolation, but none specifically have investigated the combination of these two. ${ }^{10,20-24}$ Therefore, it is still unclear whether this type of combined-mode exercise intervention achieves improvements in physical impairments affecting exercise capacity, functional outcomes or QoL in people with SCI who undertake them.

\section{Objective}

The aim of this systematic review was to investigate whether combined aerobic training and muscle strength conditioning is effective for improving the aerobic fitness, muscle strength, functional outcomes and/or QoL in people with SCI.

\section{METHODS}

\section{Eligibility criteria}

The inclusion criteria were, as follows:

Type of study: to be included, studies needed to be randomized controlled trials (RCTs), controlled trials, uncontrolled clinical trials, case series or crossover studies. For cross-over designs, studies were only included if there was a minimum of 6 weeks 'wash-out' between conditions. Theses and doctoral dissertations were not included in this systematic review. For studies that included a control arm, the control arm could be of no activity, sham exercise or regular physiotherapy treatments.

Type of participants: published studies with participants aged 18 years and older, with a SCI of traumatic or non-traumatic origin, complete or incomplete sensorimotor function (ASIA Impairment Scale grade A-D) were considered for inclusion. Only studies with participants deemed chronic SCI (that is, $>6$ months after injury) were included.

Type of interventions: to be included in this review, studies had to employ an exercise intervention that included a combination of aerobic and strength components, either in circuit training or in sequence training. Circuit training was defined as any exercise program in which a series of different exercises were arranged into components and the participant moved quickly from one component to another with a minimum recovery between them. Sequence training was defined as a single aerobic exercise modality followed by a strength mode (or vice versa), repeated not more than two times in the same session. The aerobic component had to involve exercising the large muscles of the body dynamically for a sufficient duration of time to raise exercise heart rate to a steady-state level (for example, biking, arm cranking, stepping and rowing). The strength component had to involve a voluntary contraction of muscle against a force through the use of free weights, different machines or resistive devices, with the movements controlled and carefully defined. The exercise programs needed to last for at least 3 weeks, with at least two sessions per week conducted for a minimum of $30 \mathrm{~min}$ per session.

Studies were excluded if the intervention was not typical of communitybased exercise interventions, which commonly deploy only voluntary muscle contractions. For example, studies were excluded if the interventions involved any of the following: functional electrical stimulation assisted walking, functional electrical stimulation evoked exercise or exercise with partial body weight support. These therapies are usually not available in a community-based exercise setting, thus becoming the reason for these exclusions.

Type of outcomes: to be included, studies needed to report any of the following outcome variables at baseline and post intervention: aerobic fitness (often expressed as peak oxygen uptake; $\mathrm{VO}_{2 \text { peak }}$ ), muscle strength (often expressed as a maximum weight lifted; 1RM), functional outcomes or QoL. Any assessment tool that measured these variables was included.

\section{Search strategy}

Studies were identified by searching the following electronic databases: PEDro, Web of Science, MEDLINE via OvidSP, AMED-Allied and Complementary
Medicine via OvidSP, Cinahl via Ebsco and Scopus from earliest record till February, 2013. No language limit was applied. The search combined terms covering the areas of exercise and SCI (see Supplementary Appendix 1MEDLINE search strategy). Reference lists of all the retrieved publications were also manually searched for further eligible reports if not located via bibliographic search engines.

\section{Study selection}

After eliminating duplications, the search results were screened independently by two authors of this review (VB and CO) against the eligibility criteria. Those references that could not be eliminated by title or abstract were retrieved and independently reviewed by each of the two reviewers. Disagreements were resolved by discussion or by a third reviewer (JR). Abstracts were included only if they contained all of the required information. Non-English language papers were translated. Some publications required extra information before a decision could be made on their inclusion. For those, the primary or senior authors were contacted via e-mail to provide the requisite information. Papers were discarded if the main author could not be contacted or failed to provide sufficient information about the study.

\section{Data extraction}

One reviewer extracted the following data from each included trial: (i) study design, including control condition; (ii) characteristics of trial participants, including age, gender, time since injury and body mass index; (iii) type of intervention, including type, intensity, duration and frequency of aerobic and strength components; (iv) type of outcome measure, including the aerobic and muscle strength outcomes, function and QoL measures. The second reviewer checked the extracted data. Disagreements were resolved by discussion between the two reviewers; if no agreement could be reached, a third reviewer was involved to reach consensus.

\section{Assessment of study quality}

The quality of each trial was independently assessed by two reviewers using the Physiotherapy Evidence Database (PEDro) scale. ${ }^{25}$ Any disagreements were resolved by an independent third person. The PEDro scale assesses 10 key design features important for minimizing bias and interpreting between-group differences. Higher scores reflect better-quality trials. Ratings were based on the written text and on information provided by the main authors through communication.

\section{Data analysis}

All studies provided sufficient data to enable calculation of mean difference (MD) and standardized mean difference (SMD: Hedges's $g$ ) and $95 \%$ confidence intervals (CIs). In all trials, data collected at the beginning and at the end of the intervention period were extracted. In trials that included a control arm, data were extracted for both intervention and control groups. In trials with no control group, only baseline and end of intervention data from intervention group were extracted. For RCTs or controlled trials both withingroup and between-group MD and SMD (Hedges's $g$ ) with 95\% CI were calculated for each continuous outcome. For trials with no control group, within-group MD and SMD (Hedges's $g$ ) with 95\% CI were calculated. Data were extracted from figures and authors were contacted to clarify ambiguities if necessary and feasible.

Forest plots were created using the SMD (Hedges's $g$ ) and 95\% CI to enable a visual comparison across all the studies that reported outcomes within a category (that is, aerobic outcomes, muscle strength outcomes and so on). For those outcomes that were measured homogenously across studies (that is, muscle strength and QoL), forest plots were also created using MD and $95 \%$ CI. All forest plots were created for within-group comparisons and where possible for between-group comparisons. The 95\% CI associated with the within-group and between-group MD for each outcome was used to determine whether the effect of the intervention was significant or not within a study. A meta-analysis was not appropriate due to the heterogeneity of exercise prescriptions, outcomes assessed and measurement tools of the included studies. Forest plots of within and between-group differences for all studied outcomes can be found in Figures 2-8. 


\section{RESULTS}

The original search located 7981 references for SCI. After removal of duplicates and elimination of papers based on the eligibility criteria, nine studies remained. Studies were eliminated based on the following reasons (Figure 1):

- Not a training intervention $(n=27)$

- Not an aerobic plus muscle strength intervention $(n=60)$

- Not the target population $(n=9)$

- Not the criteria intervention length $(n=3)$
- None of the criteria outcomes $(n=4)$

- Article could not be located $(n=2)$

From the nine articles that were selected, three studies were part of a single original study. ${ }^{15,17,19}$ Of these three, we discarded one because it did not match the outcome selection criteria. ${ }^{17}$ For the other two, we presented the data for the first original study ${ }^{19}$ as the second study used a subset of participants from the original one ${ }^{15}$ (personal communication with the lead author). We contacted four authors for further information. All responded and one ${ }^{14}$ provided numerical

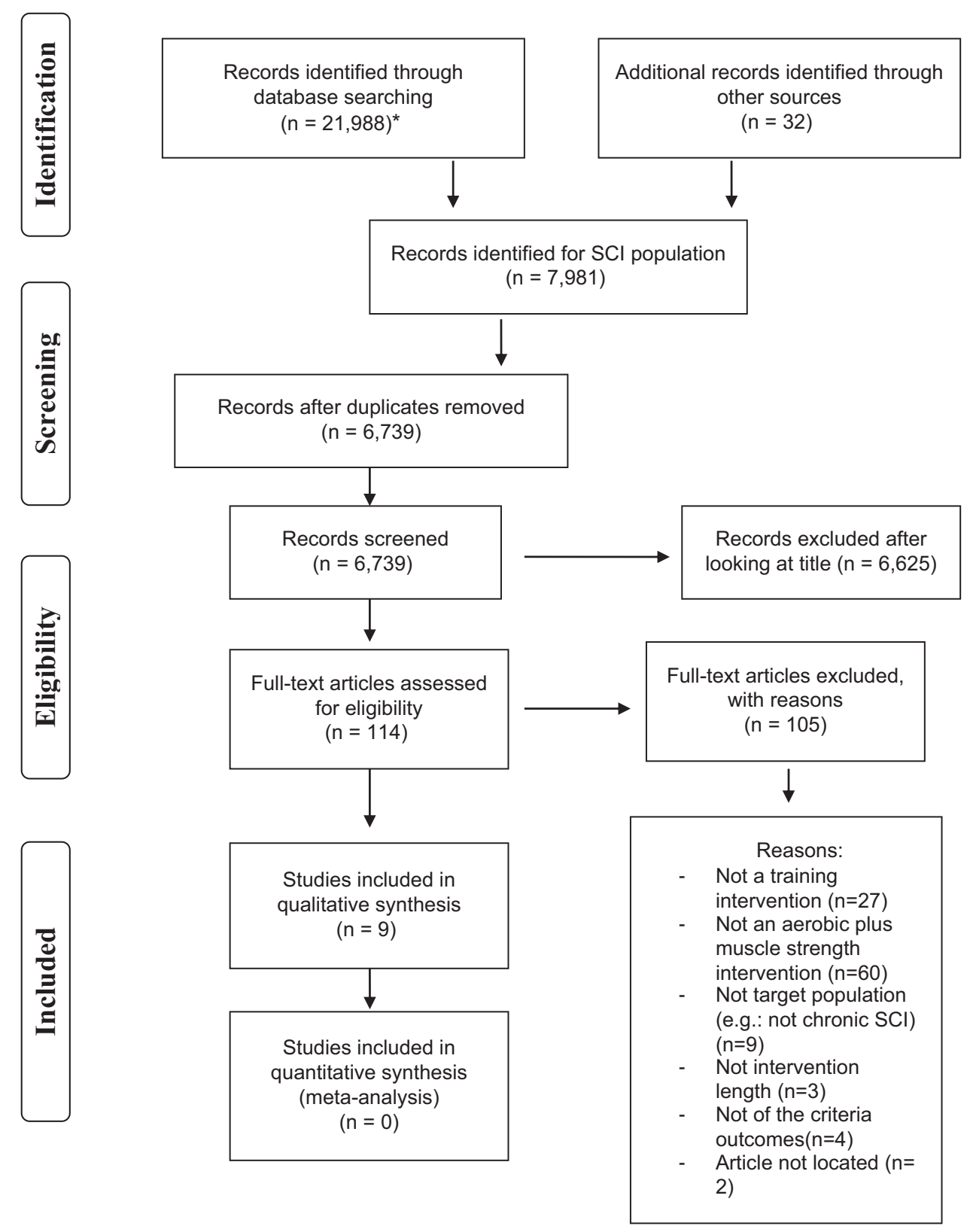

*Total number obtained after the initial search for both SCI and Stroke population.

Figure 1 Flow diagram SCl. 


\begin{tabular}{|c|c|c|c|c|c|}
\hline \multirow[t]{2}{*}{ Study name } & \multirow[t]{2}{*}{ Outcome } & \multicolumn{4}{|c|}{ Statistics for each study } \\
\hline & & $\begin{array}{l}\text { Hedges's } \\
\mathrm{g}\end{array}$ & $\begin{array}{l}\text { Lower } \\
\text { limit }\end{array}$ & $\begin{array}{l}\text { Upper } \\
\text { limit }\end{array}$ & $p$-Value \\
\hline Duran (2001) Tetraplegics & Aerobic Max PO (W) & 0.902 & 0.118 & 1.686 & 0.024 \\
\hline Jacobs $(2001,2002)$ & Aerobic VO2peak $(1 / \mathrm{m})$ & 1.532 & 0.568 & 2.497 & 0.002 \\
\hline $\begin{array}{l}\text { Keyser (2003) } \\
\text { No upper limb Impairment }\end{array}$ & Aerobic VO2peak $(\mathrm{ml} / \mathrm{m})$ & 0.032 & -0.575 & 0.640 & 0.918 \\
\hline $\begin{array}{l}\text { Keyser (2003) } \\
\text { Upper limb impairment }\end{array}$ & Aerobic VO2peak $(\mathrm{ml} / \mathrm{m})$ & 0.015 & -0.966 & 0.995 & 0.977 \\
\hline Nash (2007) Tetraplegics & Aerobic VO2peak $(1 / \mathrm{m})$ & 0.320 & -0.668 & 1.308 & 0.525 \\
\hline Sabbag (2005) & Aerobic VO2peak $(\mathrm{ml} / \mathrm{kg} / \mathrm{m})$ & 0.207 & -0.841 & 1.255 & 0.699 \\
\hline Nilsson (1975) & Aerobic VO2peak $(1 / \mathrm{m})$ & 0.391 & -0.601 & 1.382 & 0.440 \\
\hline Hicks (2003) Tetraplegics & Aerobic HR/PO relationship & 0.989 & -0.012 & 1.990 & 0.053 \\
\hline Hicks (2003) Paraplegics & Aerobic HR/PO relationship & 0.514 & -0.588 & 1.616 & 0.361 \\
\hline
\end{tabular}

\begin{tabular}{lll}
-2.00 & 0.00 & \multicolumn{1}{c}{2.00} \\
& $\begin{array}{l}\text { Got worse } \\
\text { with exercise }\end{array}$ & $\begin{array}{l}\text { Improved with } \\
\text { exercise }\end{array}$
\end{tabular}

Figure 2 Within-group SMD (Hedges's g) and 95\% Cl for aerobic fitness outcomes.

Study name
Duran (2001) Bench press
Duran (2001) Military press
Duran (2001) Butterfly press
Jacobs (2001, 2002) Military press
Jacobs (2001, 2002) Horizontal row
Jacobs (2001, 2002) Peck deck
Jacobs (2001, 2002) Preacher curls
Jacobs (2001, 2002) Latissimus pull down
Jacobs (2001, 2002) Dips (Rickshaw)
Nash (2007) Overhead press
Nash (2007) Horizontal Row
Nash (2007) Horizontal buterfly
Nash (2007) Biceps curl
Nash (2007) Latissimus dorsi pull down
Nash (2007) Triceps
Nilsson (1975) Triceps
Hicks et al (2003) Right chest
Hicks et al (2003) Left chest
Hicks et al (2003) Right biceps
Hicks et al (2003) Left biceps
Hicks et al (2003) Right anterior deltoid
Hicks et al (2003) Left anterior deltoid

\begin{tabular}{|c|c|c|c|c|}
\hline \multirow[t]{2}{*}{ Outcc } & \multicolumn{3}{|c|}{ Statistics for each study } & \multirow[b]{2}{*}{ p-Value } \\
\hline & $\begin{array}{l}\text { Difference } \\
\text { in means }\end{array}$ & $\begin{array}{l}\text { Lower } \\
\text { limit }\end{array}$ & $\begin{array}{l}\text { Upper } \\
\text { limit }\end{array}$ & \\
\hline Strength $(\mathrm{kg})$ & 19.800 & 5.425 & 34.175 & 0.007 \\
\hline Strength $(\mathrm{kg})$ & 8.300 & -5.539 & 22.139 & 0.240 \\
\hline Strength $(\mathrm{kg})$ & 11.900 & 2.136 & 21.664 & 0.017 \\
\hline Strength $(\mathrm{kg}) \#$ & 11.000 & -2.650 & 24.650 & 0.114 \\
\hline Strength $(\mathrm{kg}) \#$ & 18.000 & 2.605 & 33.395 & 0.022 \\
\hline Strength $(\mathrm{kg}) \#$ & 13.000 & -0.148 & 26.148 & 0.053 \\
\hline Strength $(\mathrm{kg}) \#$ & 3.000 & -0.099 & 6.099 & 0.058 \\
\hline Strength $(\mathrm{kg}) \#$ & 15.000 & 5.786 & 24.214 & 0.001 \\
\hline Strength $(\mathrm{kg}) \#$ & 18.000 & 8.976 & 27.024 & 0.000 \\
\hline Strength $(\mathrm{kg})$ & 20.500 & 3.984 & 37.016 & 0.015 \\
\hline Strength $(\mathrm{kg})$ & 35.500 & 26.670 & 44.330 & 0.000 \\
\hline Strength $(\mathrm{kg})$ & 23.600 & 12.721 & 34.479 & 0.000 \\
\hline Strength (kg) & 11.400 & 5.533 & 17.267 & 0.000 \\
\hline Strength (kg) & 24.100 & 12.509 & 35.691 & 0.000 \\
\hline Strength (kg) & 25.900 & 16.785 & 35.015 & 0.000 \\
\hline Strength $(\mathrm{kg})$ & 12.000 & -2.495 & 26.495 & 0.105 \\
\hline Strength $(\mathrm{kg})$ & 8.100 & -13.304 & 29.504 & 0.458 \\
\hline Strength (kg) & 9.700 & -11.027 & 30.427 & 0.359 \\
\hline Strength $(\mathrm{kg})$ & 4.300 & 0.102 & 8.498 & 0.045 \\
\hline Strength $(\mathrm{kg})$ & 4.500 & 0.934 & 8.066 & 0.013 \\
\hline Strength $(\mathrm{kg})$ & 1.500 & -1.055 & 4.055 & 0.250 \\
\hline Strength $(\mathrm{kg})$ & 1.300 & -1.378 & 3.978 & 0.341 \\
\hline
\end{tabular}

Mean difference and $95 \% \mathrm{Cl}$

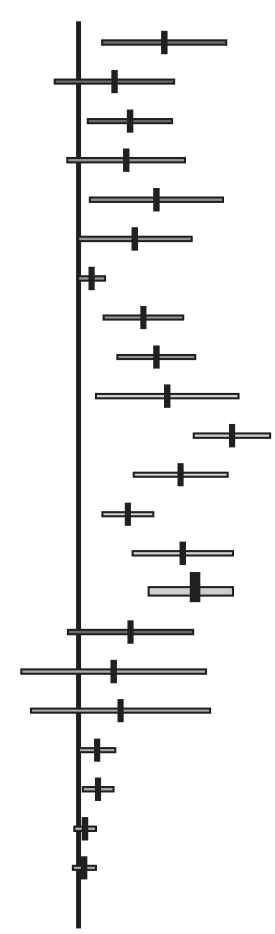

\#Values in lbs were converted to $\mathrm{kg}$

$\begin{array}{ccc}-50.00 & 0.00 & 50.00 \\ \text { Got worse with Exercise } & \text { Improved with exercise }\end{array}$

Figure 3 Within-group MD and $95 \% \mathrm{Cl}$ for muscle strength outcomes. 


\begin{tabular}{|c|c|c|c|c|c|}
\hline \multirow[t]{2}{*}{ Study name } & \multirow[t]{2}{*}{ Outcome } & \multicolumn{4}{|c|}{ Statistics for each study } \\
\hline & & $\begin{array}{l}\text { Hedges's } \\
\text { g }\end{array}$ & $\begin{array}{l}\text { Lower } \\
\text { limit }\end{array}$ & $\begin{array}{l}\text { Upper } \\
\text { limit }\end{array}$ & $p$-Value \\
\hline Duran (2001) Bench press & Strength $(\mathrm{kg})$ & 1.025 & 0.231 & 1.820 & 0.011 \\
\hline Duran (2001) Military press & Strength $(\mathrm{kg})$ & 0.447 & -0.308 & 1.201 & 0.246 \\
\hline Duran (2001) Butterfly press & Strength $(\mathrm{kg})$ & 0.907 & 0.123 & 1.692 & 0.023 \\
\hline Jacobs (2001) Military press & Strength (Ibs) & 0.668 & -0.197 & 1.532 & 0.130 \\
\hline Jacobs (2001) Horizontal row & Strength (Ibs) & 0.950 & 0.060 & 1.839 & 0.036 \\
\hline Jacobs (2001) Peck deck & Strength (lbs) & 0.789 & -0.086 & 1.663 & 0.077 \\
\hline Jacobs (2001) Preacher curls & Strength (lbs) & 0.600 & -0.260 & 1.460 & 0.171 \\
\hline Jacobs (2001) Latissimus pull down & Strength (Ibs) & 1.279 & 0.351 & 2.208 & 0.007 \\
\hline Jacobs $(2001,2002)$ Dips (Rickshaw) & Strength (Ibs) & 1.196 & 0.278 & 2.113 & 0.011 \\
\hline Nash (2007) Overhead press & Strength $(\mathrm{kg})$ & 1.217 & 0.138 & 2.297 & 0.027 \\
\hline Nash (2007) Horizontal Row & Strength $(\mathrm{kg})$ & 3.943 & 2.184 & 5.702 & 0.000 \\
\hline Nash (2007) Horizontal buterfly & Strength $(\mathrm{kg})$ & 2.128 & 0.869 & 3.386 & 0.001 \\
\hline Nash (2007) Biceps curl & Strength $(\mathrm{kg})$ & 1.906 & 0.697 & 3.114 & 0.002 \\
\hline Nash (2007) Latissimus pull down & Strength $(\mathrm{kg})$ & 2.039 & 0.801 & 3.277 & 0.001 \\
\hline Nash (2007) Triceps & Strength $(\mathrm{kg})$ & 2.787 & 1.363 & 4.211 & 0.000 \\
\hline Nilsson (1975) Triceps & Strength $(\mathrm{kg})$ & 0.865 & -0.236 & 1.965 & 0.123 \\
\hline Hicks et al (2003) Right chest & Strength $(\mathrm{kg})$ & 0.269 & -0.445 & 0.983 & 0.460 \\
\hline Hicks et al (2003) Left chest & Strength $(\mathrm{kg})$ & 0.333 & -0.383 & 1.049 & 0.362 \\
\hline Hicks et al (2003) Right biceps & Strength $(\mathrm{kg})$ & 0.728 & -0.005 & 1.461 & 0.052 \\
\hline Hicks et al (2003) Left biceps & Strength $(\mathrm{kg})$ & 0.897 & 0.153 & 1.642 & 0.018 \\
\hline Hicks et al (2003) Right anterior delto & Strength $(\mathrm{kg})$ & 0.417 & -0.301 & 1.136 & 0.255 \\
\hline Hicks et al (2003) Left anterior deltoid & Strength $(\mathrm{kg})$ & 0.345 & -0.371 & 1.061 & 0.345 \\
\hline
\end{tabular}

Hedges's $\mathrm{g}$ and $95 \% \mathrm{Cl}$

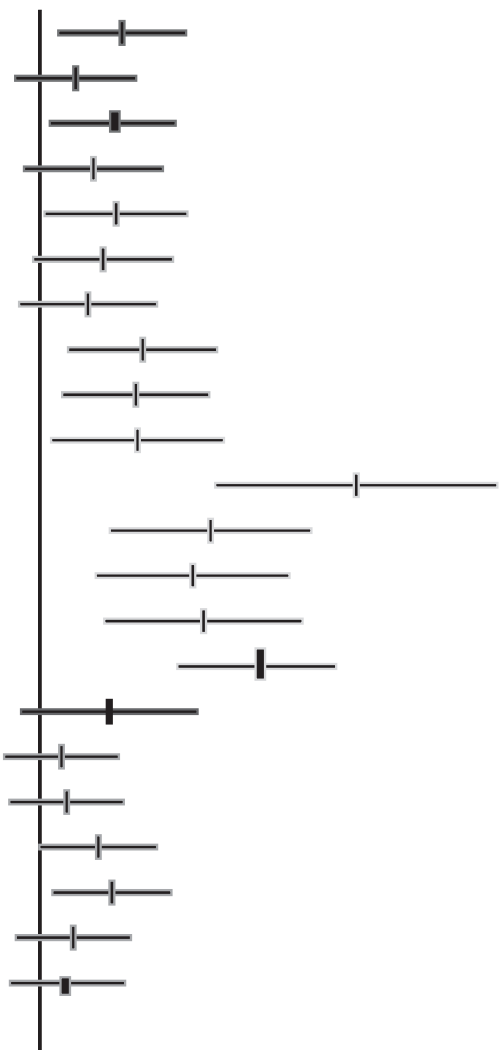

$-3.00$

Got worse with exercise

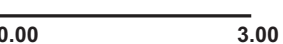

Improved with exercise

Figure 4 Within-group SMD (Hedges's $g$ ) and $95 \% \mathrm{Cl}$ for muscle strength outcome.

\begin{tabular}{lcrrrrr} 
& & \multicolumn{5}{c}{ Statistics for each study } \\
Study name & Outcome & $\begin{array}{c}\text { Difference } \\
\text { in means }\end{array}$ & $\begin{array}{l}\text { Lower } \\
\text { limit }\end{array}$ & $\begin{array}{l}\text { Upper } \\
\text { limit }\end{array}$ & p-Value \\
Hicks et al (2003) & PQOL & 5.400 & -4.123 & 14.923 & 0.266 \\
& & & & & & \\
Martin Ginis (2003) & PQOL & 2.710 & -5.167 & 10.587 & 0.500
\end{tabular}

Mean difference and $95 \% \mathrm{Cl}$

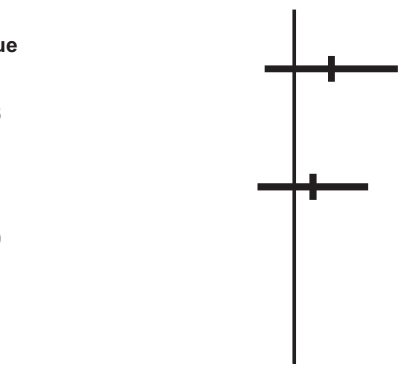

$\begin{array}{ccc}-35.00 & 0.00 & 35.00 \\ \text { Got worse with exercise } & \text { Improved with exercise }\end{array}$

Figure 5 Within-group MD and 95\% Cl for QoL outcomes.

data that were not presented in the published paper. We were unable to find any contact information for one of the author. ${ }^{26}$

\section{Characteristics of the selected publications}

Types of study. Nine studies were selected. Only two studies were RCTs published in English. ${ }^{11,14}$ One study was a case series. ${ }^{13}$ The rest of the studies analyzed measures pre and post intervention with no control group.

Participants. Study participants were both male and female, with four studies recruiting both female and male and two studies recruiting only males (Table 1). Three studies did not report gender. Lesion level 
Statistics for each study

$\begin{array}{lrcccc}\text { Study name } & \text { Outcome } & \begin{array}{c}\text { Difference } \\ \text { in means }\end{array} & \begin{array}{l}\text { Lower } \\ \text { limit }\end{array} & \begin{array}{l}\text { Upper } \\ \text { limit }\end{array} & \text { p-Value } \\ \text { Hicks et al (2003) } & \text { PQOL } & 6.000 & -3.751 & 15.751 & 0.228\end{array}$

Martin Ginis (2003) $\quad$ PQOL $\quad \begin{array}{llll}11.450 & 1.963 & 20.937 & 0.018\end{array}$
Difference in means and $95 \% \mathrm{Cl}$

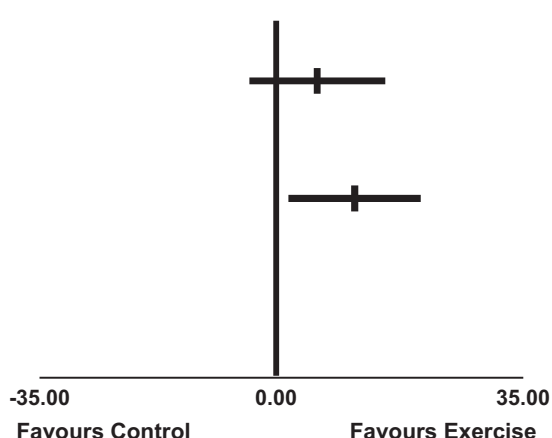

Figure 6 Between-group MD and 95\% Cl for QoL outcomes.

Statistics for each study

$\begin{array}{lccccc}\text { Study name } & \text { Outcome } & \text { Hedges's g } \begin{array}{l}\text { Lower } \\ \text { limit }\end{array} & \begin{array}{l}\text { Upper } \\ \text { limit }\end{array} & \text { p-Value } \\ \text { Hicks et al (2003) } & \text { PQOL } & 0.403 & -0.315 & 1.121 & 0.271\end{array}$

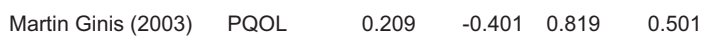

Hedges's $\mathrm{g}$ and $95 \% \mathrm{Cl}$

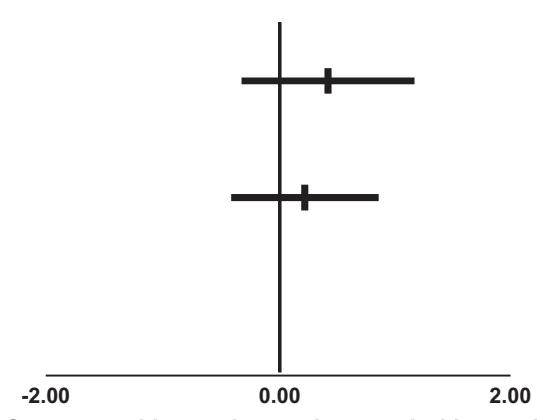

Got worse with exercise Improved with exercise

Figure 7 Within-group SMD (Hedges's g) and 95\% Cl for QoL outcomes.

Statistics for each study

$\begin{array}{lccccc}\text { Study name } & \text { Outcome } & \text { Hedges's g } & \begin{array}{l}\text { Lower } \\ \text { limit }\end{array} & \begin{array}{l}\text { Upper } \\ \text { limit }\end{array} & \text {-Value } \\ \text { Hicks et al (2003) } & \text { PQOL } & 0.416 & -0.267 & 1.098 & 0.233\end{array}$

Martin Ginis (2003) PQOL

0.815

0.11

1.518

0.023
Hedges's g and $95 \% \mathrm{Cl}$

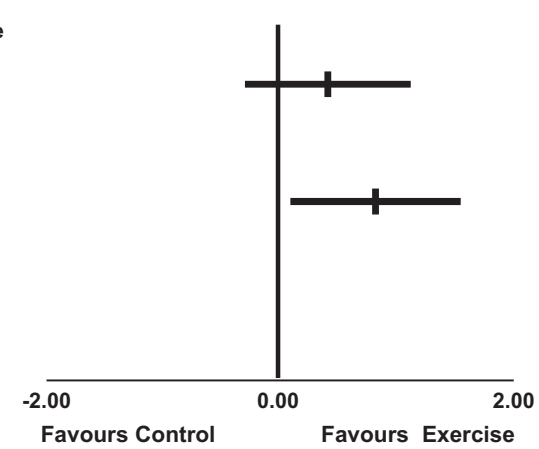

Figure 8 Between-group SMD (Hedges's g) and 95\% Cl for QoL outcomes.

ranged from $C_{4}$ to $S_{1}$. Time since injury was from 6 months till 14 years.

Types of interventions. The number of participants in each study ranged from 6 to 34. Seven studies used aerobic and muscle strength in sequence training (Table 2). The duration of the intervention ranged from 7 weeks to 9 months. Sessions were usually between 2-3 times per week and duration of the session varied from 30 to $60 \mathrm{~min}$. Only two studies used circuit training. In all studies, the aerobic component involved either arm crank ergometry or wheelchair propulsion. Five studies used arm crank ergometry. ${ }^{11,14,15,18,19,27}$ Three studies used wheelchair propulsion. ${ }^{13,16,26}$ The dose of aerobic exercise 
Table 1 Participants' characteristics

\begin{tabular}{|c|c|c|c|c|c|c|c|}
\hline Study & Sample size & Gender & $\begin{array}{l}\text { Age }(y) \text { mean } \pm \text { s.d. or } \\
\text { range }(y)\end{array}$ & Lesion level & AIS class & $\begin{array}{l}\text { Time post-injury } \\
\text { mean } \pm \text { s.d. or range }\end{array}$ & Study design \\
\hline Hicks et al. ${ }^{14}$ & $\begin{array}{l}34 \text { (Ex: } n=21 \text { Con: } \\
n=13 \text { ) }\end{array}$ & $\begin{array}{l}23 \text { male/ } 11 \\
\text { female }^{a}\end{array}$ & $\begin{array}{l}\text { Ex: } 36.9 \pm 11.4 \\
(19-65) \\
\text { Con: } 43.2 \pm 9.3 \\
(29-63)\end{array}$ & $\begin{array}{l}\text { Ex: } \\
\text { Tetra: } n=11\left(\mathrm{C}_{4}-\mathrm{T}_{2}\right) \\
\text { Para: } n=10\left(\mathrm{~T}_{3}-\mathrm{S}_{1}\right) \\
\text { Con: } \\
\text { Tetra: } n=7\left(\mathrm{C}_{4}-\mathrm{C}_{7}\right) \\
\text { Para: } n=6\left(\mathrm{~T}_{7}-\mathrm{L}_{2}\right)\end{array}$ & $\begin{array}{l}\text { Ex: } \\
\text { A: } n=6 \\
\text { B: } n=3 \\
\text { C: } n=6 \\
\text { D: } n=6 \\
\text { Con: } \\
\text { A: } n=7 \\
\text { B: } n=0 \\
\text { C: } n=3 \\
\text { D: } n=3\end{array}$ & $\begin{array}{l}\text { Ex: } \\
7.7 \pm 6.4 \\
(1-22) \\
\text { Con: } \\
12.1 \pm 7.3 \\
(3-24) \\
\text { (y) }\end{array}$ & $\begin{array}{l}\text { Randomized } \\
\text { controlled trial }\end{array}$ \\
\hline $\begin{array}{l}\text { Martin Ginis } \\
\text { et al. } 11 \mathrm{~b}\end{array}$ & $\begin{array}{l}34 \text { (Ex: } n=21 \\
\text { Con: } n=13 \text { ) }\end{array}$ & $\begin{array}{l}23 \text { male/ } 11 \\
\text { female }\end{array}$ & $38.6 \pm 11.7$ & & $\begin{array}{l}\text { Complete: } 14 \\
\text { Incomplete: } 13\end{array}$ & $\begin{array}{l}10.4 \pm 11.79 \\
\text { (y) }\end{array}$ & $\begin{array}{l}\text { Randomized con- } \\
\text { trolled trial }\end{array}$ \\
\hline Duran et al. ${ }^{13}$ & 13 & $\begin{array}{l}12 \text { male/ } 1 \\
\text { female }\end{array}$ & $26.3 \pm 8.3$ & $\begin{array}{l}\mathrm{T}_{3}-\mathrm{T}_{6}: n=4 \\
\mathrm{~T}_{6}-\mathrm{T}_{12}: n=9\end{array}$ & $\begin{array}{l}\text { A: } n=11 \\
\text { B: } n=1 \\
\text { C: } n=1\end{array}$ & 10 (months) & Case series \\
\hline $\begin{array}{l}\text { Jacobs } \\
\text { et al. }{ }^{15 \mathrm{c}}\end{array}$ & 6 & & $35.7 \pm 9.8$ & $\mathrm{~T}_{5}-\mathrm{T}_{12}$ & & $6.0 \pm 4.7$ (months) & Repeated measures \\
\hline Jacobs et al. ${ }^{19}$ & 10 & & $39.4 \pm 6.0$ & $\mathrm{~T}_{5}-\mathrm{L}_{1}$ & & $7.3 \pm 6.0$ (months) & Repeated measures \\
\hline Keyser et al. ${ }^{16}$ & $27(17 \mathrm{SCl})^{\mathrm{d}}$ & & $41 \pm 10$ & $\begin{array}{l}\text { No upper limb impair- } \\
\text { ment } n=20 \\
\text { Upper limb impairment: } \\
n=7\end{array}$ & & & Repeated measures \\
\hline Nash et al. ${ }^{18}$ & 7 & Male & $35-58$ & $\mathrm{~T}_{5}-\mathrm{T}_{12}$ & $A$ and $B$ & $13.1 \pm 6.6(y)$ & Repeated measures \\
\hline $\begin{array}{l}\text { Sabbag } \\
\text { et al. }\end{array}$ & $\begin{array}{l}\text { Ex: } 6 \mathrm{SCl} \\
\text { Con: } 6 \text { healthy } \\
\text { sedentary }\end{array}$ & Male & $\begin{array}{l}\text { Ex: } 31.5 \pm 10.5 \\
\text { Con: } 31.3 \pm 10.2\end{array}$ & $\mathrm{~T}_{1}$ and below & & $1(y)$ & Repeated measures \\
\hline $\begin{array}{l}\text { Nilsson } \\
\text { et al. }\end{array}$ & $\begin{array}{l}12 \text { (11 SCl, } 1 \\
\text { poliomyelitis) }\end{array}$ & & $36(22-55)$ & $\begin{array}{l}\mathrm{C}_{6}-\mathrm{C}_{7}: 1 \\
\mathrm{C}_{7}-\mathrm{T}_{1}: 1 \\
\mathrm{~T}_{4}: 1 \\
\mathrm{~T}_{11}: 1 \\
\mathrm{~T}_{11}-\mathrm{T}_{12}: 3 \\
\mathrm{~T}_{12}: 3 \\
\mathrm{~T}_{12}-\mathrm{L}_{1}: 2\end{array}$ & $\begin{array}{l}\text { Complete: } 10 \\
\text { Incomplete: } 2\end{array}$ & $10(4-19)(y)$ & Repeated measures \\
\hline
\end{tabular}

Abbreviations: Con, control; Ex, exercise; SCI, spinal cord injury; Sup, supervised; Unsup, unsupervised.

${ }^{a}$ Not reported, but presumed from Martin Ginis et al. ${ }^{11}$

${ }^{b}$ Part of the same population from another study, same participants from Hicks et al. ${ }^{14}$ data after 3-month intervention.

'This study used a subset of participants from Jacobs et al. ${ }^{19}$ therefore the outcomes are reported from Jacobs et al. ${ }^{19}$ only.

dNo information provided from the $17 \mathrm{SCl}$ participants.

was progressively increased from 15 to $40 \mathrm{~min}$ for duration and maintaining an intensity of between 40 and $70 \%$ of peak heart rate $\left(\mathrm{HR}_{\text {peak }}\right)$ in most of the studies.

Types of outcomes. For the muscle strength component a variety of exercises involving the main muscles of the upper body were performed using weight machines and elastic bands. Examples of the exercises were the military press, latissimus dorsi pull-down, horizontal row and biceps curls. ${ }^{28}$

\section{Methodological quality}

The quality of studies was based on the PEDro scale. Studies scoring 9-10 on PEDro scale are considered of 'excellent' quality, scores ranging from 6 to 8 are considered of 'good' quality. Scores ranging from 4 to 5 are of 'fair' quality and finally studies, where scores are below 4 are of 'poor' quality. ${ }^{29}$

Only one RCT scored in each of the 'fair' 14 and 'good'11 categories based on the PEDro scale. The rest of the studies scored between 0 and 3 on the PEDro scale, showing a 'poor' level of quality. None of the studies utilized concealed allocation and blinding of all participants, therapists and all assessors. Two studies specified the eligibility criteria. ${ }^{11,13}$ Two studies allocated subjects randomly, had baseline similarity and reported between-group statistics. ${ }^{11,14}$ Another two studies analyzed the 'intention to treat'. ${ }^{11,26}$ Four studies measured key outcomes in more than $85 \%$ of the initial subjects. ${ }^{11,14,18,26}$ Finally, five studies provided point measures and measures of variability for at least one key outcome. ${ }^{11,14,16,18,26}$

\section{Study outcomes}

All studies provided sufficient data to enable calculation of MD and SMD (Hedges's $g$ ) and 95\% CI for outcomes related to aerobic fitness, muscle strength and QoL. None of the included studies described any functional or physical activity outcomes. The aerobic outcome most often reported was $\mathrm{VO}_{2}$ peak, with the exception of one study that used the exercise relationship between HR and power output during an arm crank ergometry assessment. ${ }^{14}$ The most common outcome measure for muscle strength was weight lifted by each muscle group in kilograms (kg). All of the studies used 1-Repetition Maximum 


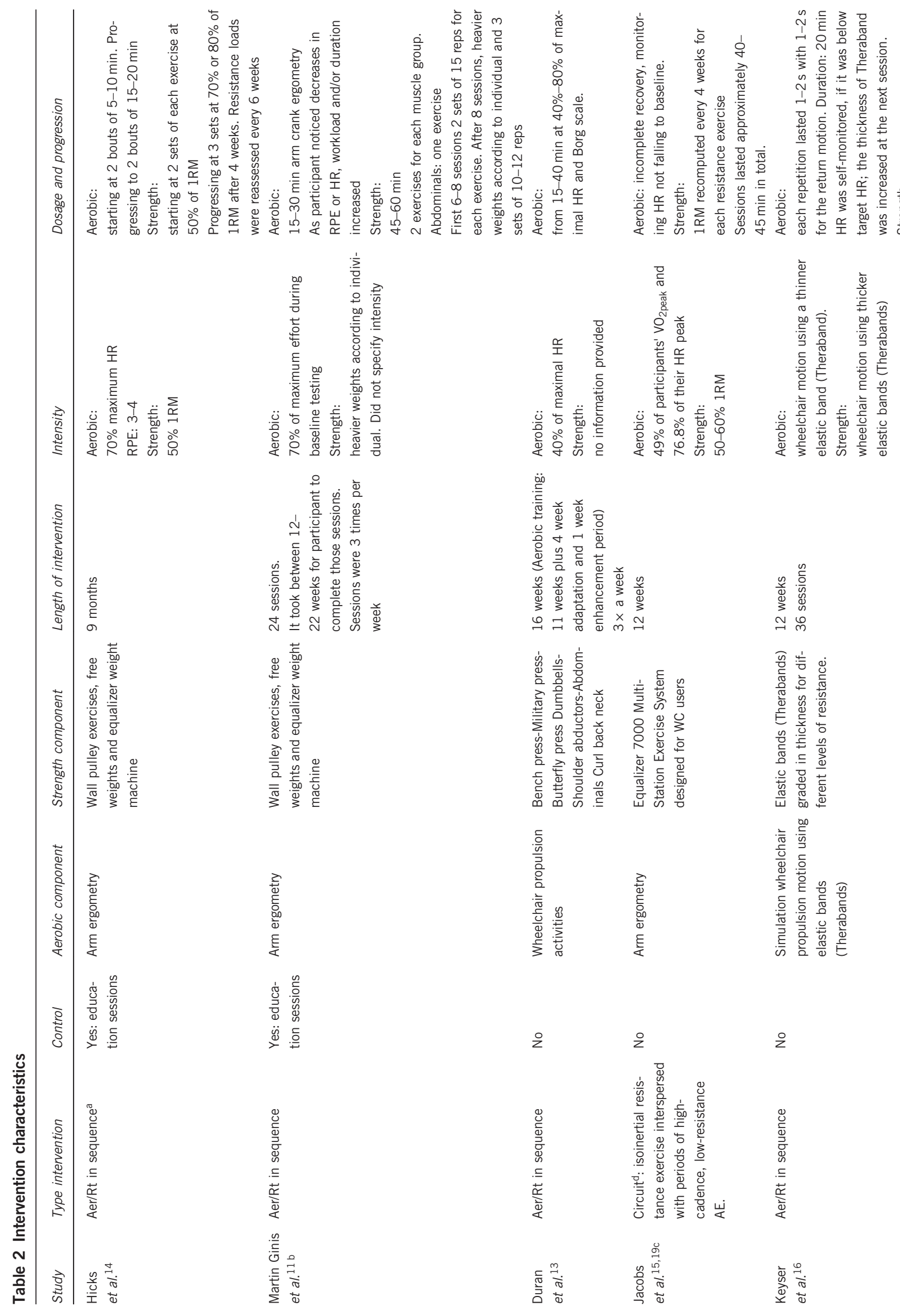

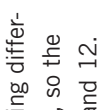
ज.

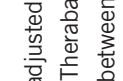

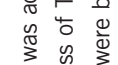

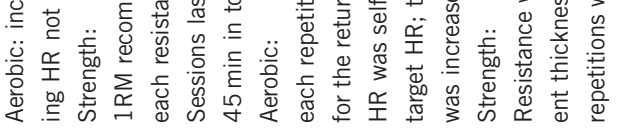

岗它
产市

2 


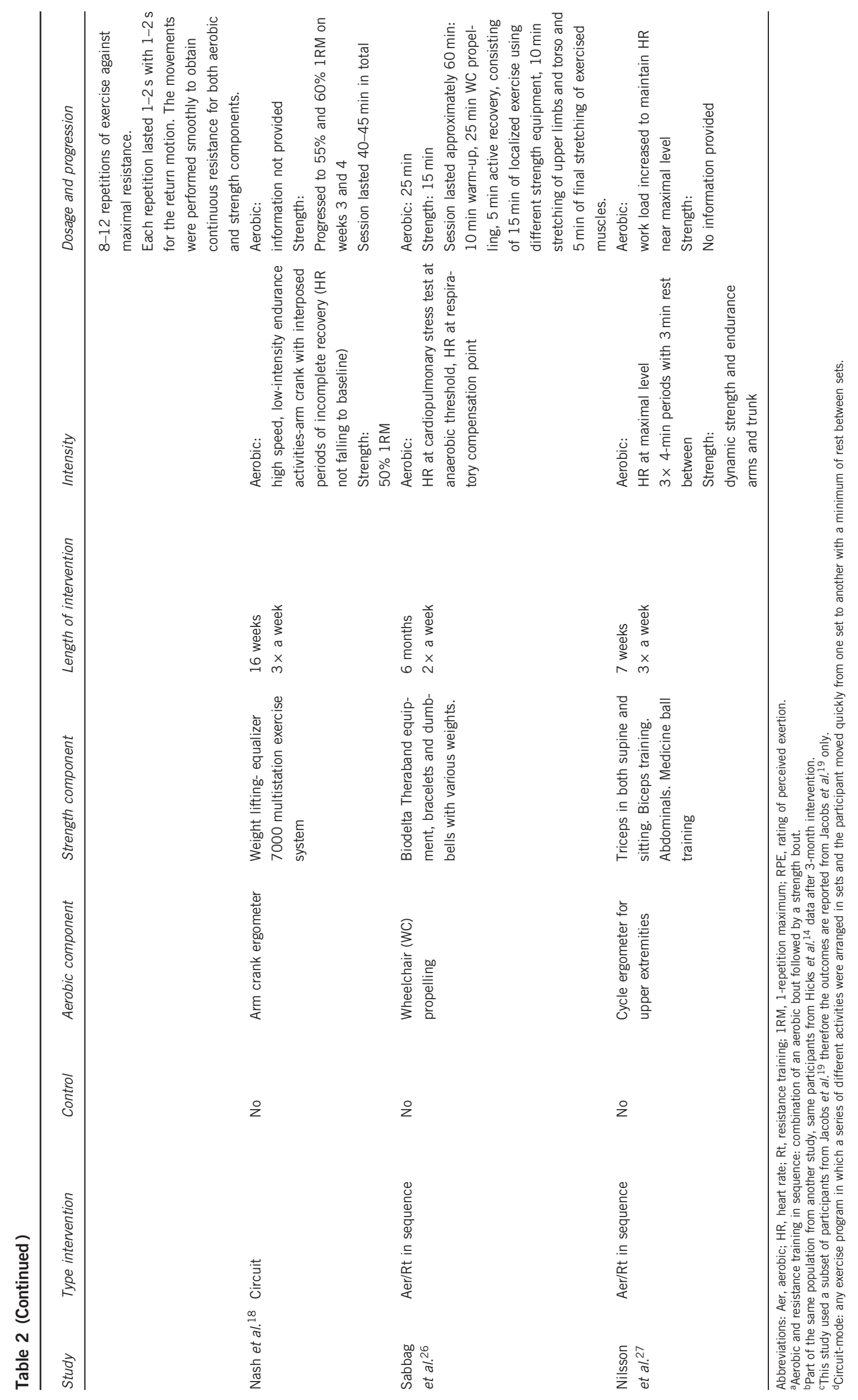


(1RM) in kilograms to express muscle strength outcomes. One study used an imperial measure of weight (pounds), but this was converted to kilograms for the presentation in the forest plot. ${ }^{19}$

\section{Aerobic fitness outcomes}

Seven studies reported aerobic outcomes. One of the RCT studies of 'fair' quality that used a twice weekly circuit resistance in combination with arm ergometry intervention, showed a significant within-training group effect on aerobic fitness with MD (95\% CI) of 13.8 b.p.m. W $\mathrm{W}^{-1}$ (0.63-26.9) and between-group effect on aerobic fitness with MD (95\% CI) value of 13.1 b.p.m. $\mathrm{W}^{-1}(0.2-25.98)$ but only for participants with tetraplegia (Table 3$){ }^{14}$

Four studies of poor quality and with no control groups found no statitistically significant within-group improvements in aerobic measures. ${ }^{16,18,26,27}$ The MD (95\% CI) from Keyser et al. was 11.0 $\mathrm{ml} \mathrm{min}{ }^{-1}$ (-197.6 to 219.6) for participants with no upper limb impairment and $5.0 \mathrm{ml} \mathrm{min}{ }^{-1}$ (-332.34 to 342.34) for participants with upper limb impairment. The MD (95\% CI) from Nash et al. was $0.171 \mathrm{~min}^{-1}$ ( -0.35 to 0.69$)$. The MD (95\% CI) from Nilsson et al. was $1.0 \mathrm{ml} \mathrm{kg}^{-1} \mathrm{~min}^{-1}(-4.05$ to 6.05$)$ and the $\mathrm{MD}$ (95\% CI) from Sabbag et al. was $0.191 \mathrm{~min}^{-1}$ ( -0.28 to 0.66 ).

One study of 'poor' quality showed a statistically significant withingroup difference in aerobic fitness, with a MD (95\% CI) of $20 \mathrm{~W}$ (3.49-36.5) and a SMD (95\% CI) of $0.90(0.11-1.68) .{ }^{13}$ Another study showed also a statistically significant within-group difference, with a MD (95\% CI) of $0.431 \mathrm{~min}^{-1}(0.19-0.66)$ and a SMD (95\% CI) of $1.53(0.56-2.49)(19)$. In this case the dose for aerobic exercise was reported, which was progressively increasing in duration from 15 to $40 \mathrm{~min}$ and from 40 to $80 \%$ of $\mathrm{HR}_{\max }$. Figure 2 shows the forest plot for aerobic fitness outcomes.

\section{Muscle strength outcomes}

There is some evidence regarding positive muscle strength outcomes (Table 4). Five studies investigated the effectiveness of exercise training on muscle strength. Most of these studies showed statistically significant within-group differences in muscle strength on some of the muscle groups assessed.

One study ${ }^{18}$ had significant within-group differences for the following upper limb exercises: horizontal row exercise with a MD (95\% CI) of $35.5 \mathrm{~kg}$ (26.6-44.3); overhead press with a MD (95\% CI) of $20.5 \mathrm{~kg}$ (3.9-37.01); horizontal butterfly (pectoralis major and minor) with a $\mathrm{MD}(95 \% \mathrm{CI})$ of $23.6 \mathrm{~kg}$ (12.7-34.4); biceps curl with a $\mathrm{MD}(95 \% \mathrm{CI})$ of $11.4 \mathrm{~kg}$ (5.5-17.2); latissumus dorsi pull-down with a $\mathrm{MD}(95 \% \mathrm{CI})$ of $24.1 \mathrm{~kg}(12.5-35.6)$; and triceps extension with a MD (95\% CI) of $25.9 \mathrm{~kg}(16.78-35.01)$.

Some statistically significant within-group differences were found in another study of 'poor' quality for most of the muscle strength outcomes. The most distinctive results in this case, were found on triceps dips with a MD (95\% CI) of $39.0 \mathrm{lb}(11.61-66.3)$ horizontal row with $\mathrm{MD}(95 \% \mathrm{CI}) 40.4 \mathrm{lb}(4.6-76.1)$ and latissumus dorsi pulldown with MD (95\% CI) $33.2 \mathrm{lb}$ (11.4-54.9). ${ }^{19}$

The only 'fair' quality study that used a control group reported statistically significant improvements in muscle strength only on one of the muscle groups assessed. In this case, the within-group MD (95\% CI) was $4.5 \mathrm{~kg}(0.93-8.06)$ and $4.3 \mathrm{~kg}$ (0.10-8.49), for right and left biceps, respectively. A significant between-group effect was found for right biceps only with a MD (95\% CI) of $4.6 \mathrm{~kg}$ (0.38-8.8). ${ }^{14}$ Figures 3 and 4 show the forest plots for muscle strength outcomes.

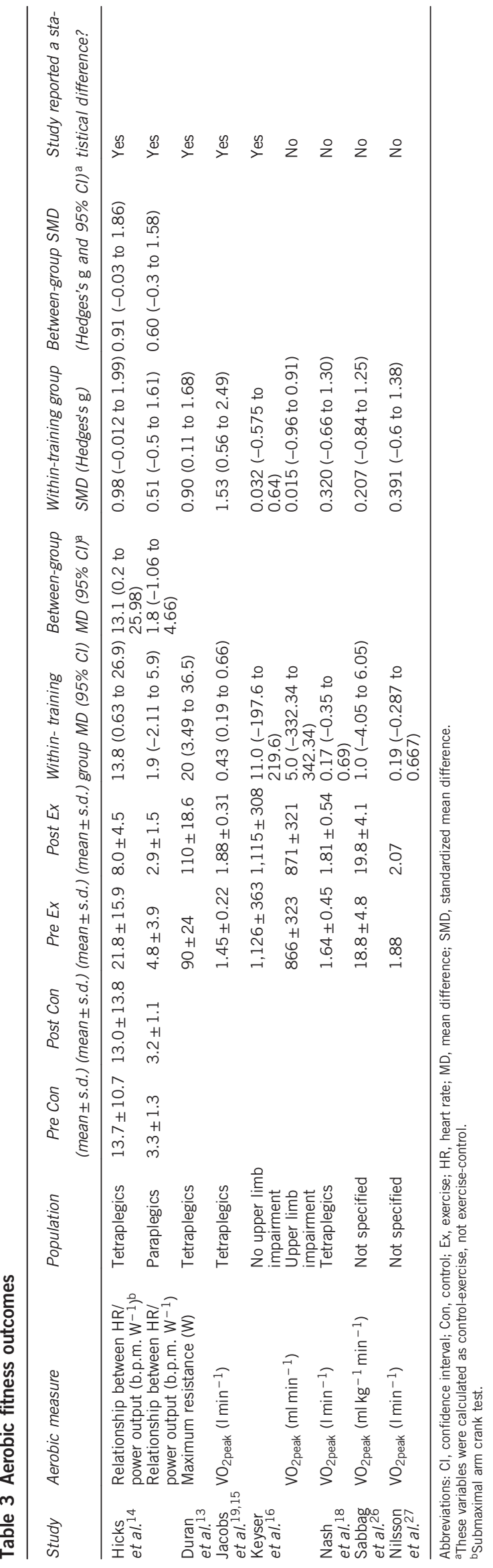




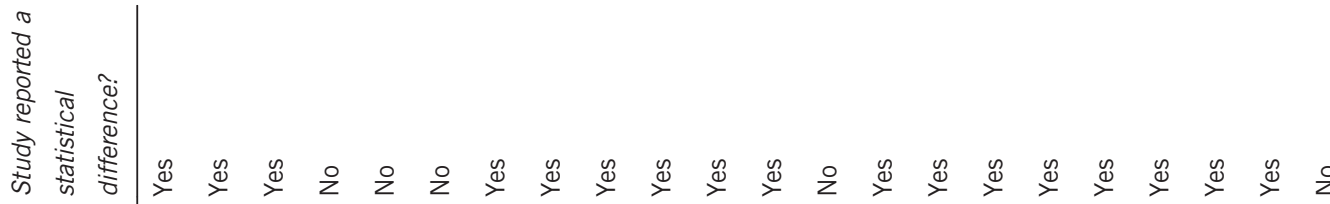




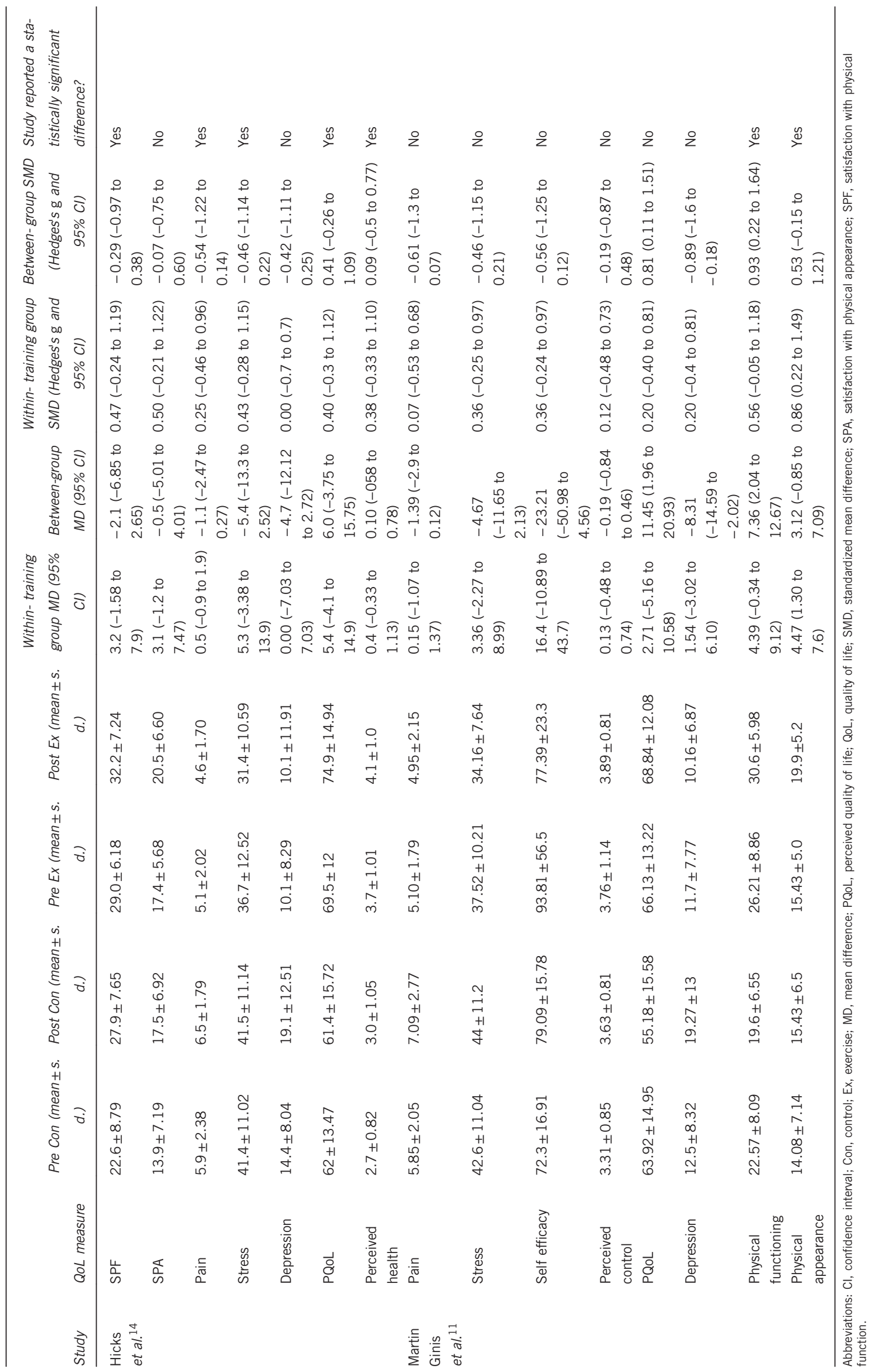




\section{Quality of life (QoL) outcomes}

Two studies looked at QoL, using different questionnaires to obtain results regarding pain perception, symptom self-efficacy, perceived control, stress, satisfaction with fundamental needs of daily living, perceived health and depression (Table 5). ${ }^{11,14}$ The heterogeneity of the scales used to measure QoL made it difficult to compare the studies. One of the questionnaires used was the Reboussin et al. 9-item body satisfaction questionnaire, ${ }^{30}$ which included Physical Appearance and Physical Functioning. Only one of studies included in this review found a significant effect on physical appearance using the body satisfaction questionnaire, but only for within-group differences with a MD (95\% CI) of $4.47(1.30-7.6){ }^{14}$ The only between-group significant difference was found for physical functioning, with a MD (95\% CI) of 7.36 (2.04-12.67). ${ }^{14}$ The Perceived Quality of Life Scale (PQoL) was used by two studies. One study found a statistically significant between-group difference with a MD (95\% CI) of 11.45 (1.96-20.93) but the other study did not find a significant effect in the same group of participants, as the MD (95\% CI) was of 6.0 ( -3.75 to 15.75). Neither study found a statistically significant within-group difference with a MD $(95 \% \mathrm{CI})$ of $5.4(-4.1$ to 14.9$)$ and $2.71(-5.16$ to 10.5$)$, respectively. ${ }^{11,14}$ Results within-group and between-groups for outcomes such as pain, stress and depression were not significant in either study. ${ }^{8,12}$ See Figures 5-8 for forest plots for QoL outcomes.

\section{DISCUSSION}

Overall, the quality of the published evidence surveyed in this review was not sufficiently robust to determine the effectiveness of combined aerobic training and muscle strength conditioning for improving aerobic, muscle strength, QoL and functional outcomes. Some significant positive effects were found for aerobic fitness outcomes, after resistance training was combined with arm crank exercise in a study of 'fair' PEDro-denoted quality. However, those results were found in one population only (tetraplegics), so there was little evidence to draw any firm conclusions from this one study. ${ }^{14}$ Any other significant findings were noted in studies of poor quality. ${ }^{13,19}$ Muscle strength showed some significant positive outcomes on some of the upper limb muscles assessed, but those results were not consistent, showing only within-group differences on some specific muscles. The 'fair' PEDro denoted quality study showed a significant effect for within- and between- groups changes but only on one specific muscle (right biceps). Our findings of low quality levels of evidence among studies related to exercise interventions in people after SCI were similar to findings of other systematic reviews that looked at the effects of exercise training on physical capacities and functional performance in people with SCI. ${ }^{10,23,31}$

Given our muscle strength results, it seems that the ideal dose would be $50-80 \% 1 \mathrm{RM}$ with progression applied to maintain overload adaptation. ${ }^{14}$ It is interesting to highlight that in one study that showed improvements in muscle strength, the strength training dose started with $50 \% 1 \mathrm{RM}$ with the number of sets of exercises also increasing over time. ${ }^{14}$ In regards to studies that did not specify a dose, the reason for the inconclusive results could have been that they used light resistance that was subthreshold for a training effect. The other two studies that found significant effects on some of the muscles assessed also specified a training dose starting at $50 \%$ of $1 \mathrm{RM}$ and increasing progressively up to $60 \% 1 \mathrm{RM} .{ }^{18,19}$ The rest of the studies did not specify an appropriate dose of exercise. ${ }^{13,27}$

Making comparisons between studies and drawing any conclusions about QoL outcomes is problematic due to the lack of one standard scale and the use of many psychological scales and questionnaires. It has been stated in previous studies that a more concrete and universal definition of QoL is necessary to make a true comparison between studies. $^{32}$ The evidence regarding QoL is inconclusive, as only three out of fifteen outcomes showed positive results towards improvements in that domain. Overall, evidence is insufficient to draw a solid conclusion regarding QoL outcomes. ${ }^{11,14}$

In regards to the general poor quality of the studies, there are some aspects that are worth mentioning. Among aspects that are missing from SCI studies are blinding of subjects and therapists. These missing aspects are due to difficulties in achieving blinding when using exercise interventions. Another aspect missing in most of the studies is specifying the criteria for selection of participants, this is explained by the fact that narrow criteria could lead to low number of participants and most studies tend to use a pool of participants that could be recruited easily from a rehabilitation center or hospital, rather than recruiting participants with SCI from different sites. Having found only two RCT studies that matched our criteria, it could be implied that performing RCT among the SCI population is difficult to accomplish. There is one barrier when working with people with SCI that would make conducting RCT challenging. An individual with a SCI, when being offered a high quality training intervention or being part of a control group, would most likely refuse to take part if allocated to the control group; there are ethical concerns with denying the possibility of some kind of treatment by including a control group in the study. ${ }^{33}$

Our inconclusive findings in aerobic fitness, muscle strength and QoL outcomes were similar to the ones identified in previous systematic reviews about physical interventions in people with SCI. ${ }^{10,23,32}$ The differences between our review and those previously published were that (i) our review searched only for people with SCI in a chronic state (more than six months after injury), as we wanted to avoid any influence of natural neurological recovery that happens during the first six months after the injury; and (ii) our review only looked at interventions involving either circuit training or aerobic plus muscle strength training performed in sequence, because we were interested in voluntary exercises that are commonly used in community-based exercise interventions, without the use of any special equipment or devices, such as a treadmill or functional electrical stimulation. ${ }^{10,23,32}$

Finally, our search criteria did not limit the spinal cord injury level because that would have limited the number of research studies and missed relevant information for this systematic review. Although an aerobic training effect would have not been expected in people with higher lesion levels, a significant effect for aerobic fitness was found in tetraplegics.

\section{Limitations}

A limitation of this review was that we excluded thesis and doctoral dissertations, so we may have missed some unpublished studies with valuable data. Another limitation was that some full text articles could not be located, so we could only use the information provided by title and abstract to make the selection of the articles.

\section{CONCLUSION}

Overall, the existing literature on the spinal cord injured population relating to the effects of combined aerobic and muscle strength training on aerobic fitness, muscle strength, function and QoL is scarce and of low quality. There is little evidence to support any statement in favor of aerobic fitness improvements after combined aerobic and muscle strength training. The results of this systematic review provide initial evidence of significant improvements in muscle strength after a combined aerobic and muscle strength training. 
However, these results are insufficient to draw any definite conclusions. The only conclusion that can be drawn is that the ideal dose for muscle strength training would be $50-80 \% 1 \mathrm{RM}$ with progression applied. As far as QoL is concerned, although the two studies that analyzed this outcome were of 'fair' and 'good' quality, results were limited and lacked consistency for both within and between-groups differences. Functional outcomes were not described in any of the selected studies. Therefore, conclusions about the effectiveness of combined aerobic and muscle strength training on QoL and function cannot be made.

To advance this field of research, further RCT with larger numbers of participants are needed to make a definite conclusion about the influence of combined aerobic and muscle strength training on aerobic fitness, muscle strength and QoL in people with SCI. It would also be of great importance to include functional assessments in within the RCT as part of the main outcome.

\section{DATA ARCHIVING}

There were no data to deposit.

\section{CONFLICT OF INTEREST}

The authors declare no conflict of interest.

1 Beninato M, O'Kane KS, Sullivan PE. Relationship between motor FIM and muscle strength in lower cervical-level spinal cord injuries. Spinal Cord 2004; 42: 533-540.

2 Ferreira VMD, Varoto R, Cacho EWA, Cliquet A. Relationship between function, strength and electromyography of upper extremities of persons with tetraplegia. Spinal Cord 2012; 50: 28-32.

3 Harvey L. Strength Training. Management of Spinal Cord Injuries. Churchill Livingstone: Edinburgh, UK, 2008; 155-176.

4 Harvey L. (ed) Background information. Management of Spinal Cord Injuries. Churchill Livingstone: Edinburgh, 2008; 3-33.

5 Phillips WT, Kiratli BJ, Sarkarati M, Weraarchakul G, Myers J, Franklin BA et al. Effect of spinal cord injury on the heart and cardiovascular fitness. Curr Probl Cardiol 1998; 23: 641-716.

6 Maggioni MA, Ferratini M, Pezzano A, Heyman JE, Agnello L, Veicsteinas et al. Heart adaptations to long-term aerobic training in paraplegic subjects: an echocardiographic study. Spinal Cord 2012; 50: 538-542.

7 Shields RK. Muscular, skeletal, and neural adaptations following spinal cord injury. J Orthop Sports Phys Ther 2002; 32: 65-74.

8 Noreau L, Shephard R. Spinal cord injury, exercise and quality of life. Sports Med 1995; 20: 226-250.

9 Noreau L, Shephard RJ, Simard C, Pare G, Pomerleau P. Relationship of impairment and functional ability to habitual activity and fitness following spinal cord injury. Int $J$ Rehabil Res 1993; 16: 265-275.

10 Harvey LA, Lin CWC, Glinsky JV, De Wolf A. The effectiveness of physical interventions for people with spinal cord injuries: a systematic review. Spinal Cord 2009; 47: $184-195$.

11 Martin Ginis KA, Latimer AE, McKechnie K, Ditor DS, McCartney N, Hicks AL et al. Using exercise to enhance subjective well-being among people with spinal cord injury: the mediating influences of stress and pain. Rehabil Psychol 2003; 48: 157-164.
12 Schonherr MC, Groothoff JW, Mulder GA, Eisma WH. Prediction of functional outcome after spinal cord injury: a task for the rehabilitation team and the patient. Spinal Cord 2000; 38: 185-191.

13 Duran FS, Lugo L, Ramirez L, Lic E. Effects of an exercise program on the rehabilitation of patients with spinal cord injury. Arch Phys Med Rehabil 2001; 82: 1349-1354.

14 Hicks AL, Martin KA, Ditor DS, Latimer AE, Craven C, Bugaresti J et al. Long-term exercise training in persons with spinal cord injury: effects on strength, arm ergometry performance and psychological well-being. Spinal Cord 2003; 41: 34-43.

15 Jacobs PL, Mahoney ET, Nash MS, Green BA. Circuit resistance training in persons with complete paraplegia. J Rehabil Res Dev 2002; 39: 21-28.

16 Keyser RE, Rasch EK, Finley M, Rodgers MM. Improved upper-body endurance following a 12-week home exercise program for manual wheelchair users. J Rehabil Res Dev 2003; 40: 501-510.

17 Nash MS, Jacobs PL, Mendez AJ, Goldberg RB. Circuit resistance training improves the atherogenic lipid profiles of persons with chronic paraplegia. J Spinal Cord Med 2001; 24: 2-9.

18 Nash MS, van de Ven I, van Elk N, Johnson BM. Effects of circuit resistance training on fitness attributes and upper-extremity pain in middle-aged men with paraplegia. Arch Phys Med Rehabil 2007; 88: 70-75.

19 Jacobs PL, Nash MS, Rusinowski JW. Circuit training provides cardiorespiratory and strength benefits in persons with paraplegia. Med Sci Sports Exerc 2001; 33: 711-717.

20 Davis F, Kumaran DS, Nair S, Vijayamuniraj J. Physical fitness training for people with spinal cord injury (Protocol). Cochrane Database Syst Rev 2009, Issue 3. Art. No.: CD007955. DOI: 10.1002/14651858.CD007955.

21 Devillard X, Rimaud D, Roche F, Calmels $P$. Effects of training programs for spinal cord injury. Ann Readapt Med Phys 2007; 50: 490-498.

22 Hicks AL, Pelletier CA, Ditor DS, Foulon B, Wolfe DL. The effects of exercise training on physical capacity, strength, body composition and functional performance among adults with spinal cord injury: a systematic review. Spinal Cord 2011; 49: 1103-1127.

23 Valent L, Dallmeijer A, Houdijk H, Talsma E, van der Woude L. The effects of upper body exercise on the physical capacity of people with a spinal cord injury: a systematic review. Clin Rehabil 2007; 21: 315-330.

24 Haisma JA, van der Woude LHV, Stam HJ, Bergen MP, Sluis TAR. Bussmann JBJ. Physical capacity in wheelchair-dependent persons with a spinal cord injury: a critical review of the literature. Spinal Cord 2006; 44: 642-652.

25 Moseley AM, Herbert RD, Sherrington C, Maher CG. Evidence for physiotherapy practice: A survey of the Physiotherapy Evidence Database (PEDro). Aust J Physiother 2002; 48: 43-49.

26 Sabbag LMS, Mourao MA, Saito ET, Yasbek P, Matias EA, Cardoso CV et al. Comparison of supervised physical conditioning effects in patients with spinal cord injury and normal sedentary individuals. 40128. Medimond SRL: Bologna, 2005; 973-976.

27 Nilsson S, Staff PH, Pruett ED. Physical work capacity and the effect of training on subjects with long-standing paraplegia. Scand J Rehabil Med 1975; 7: 51-56.

28 Mudge S. A growing web resource of physiotherapy exercises. Aust J Physiother 2008; 54: 225 .

29 Straus SE, Richardson WS., Glasziou P, Haynes RB. Evidence-Based Medicine: How to Practice and Teach EBM. Elsevier Churchill Livingstone: Toronto, Canada, 2005.

30 Reboussin BA, Rejeski WJ, Martin KA, Callahan K, Dunn AL, King AC et al. Correlates of satisfaction with body function and body appearance in middle- and older aged adults: The Activity Counseling Trial (ACT). Psychol Health 2000; 15: 239-254.

31 Hicks AL, Ginis KAM, Pelletier CA, Ditor DS, Foulon B, Wolfe DL. The effects of exercise training on physical capacity, strength, body composition and functional performance among adults with spinal cord injury: a systematic review. Spinal Cord 2011: 49: 1103-1127.

32 Hill MR, Noonan VK, Sakakibara BM, Miller WC, Team SR. Quality of life instruments and definitions in individuals with spinal cord injury: a systematic review. Spinal Cord 2010; 48: 438-450.

33 World Medical Organization Declaration of Helsinki 2008. Available from http://www. wma.net/e/ethicsunit/helsinki.htm.

Supplementary Information accompanies this paper on the Spinal Cord website (http://www.nature.com/sc) 